\title{
Prevention of Splenic Injury During Colonoscopy by Positioning of the Patient
}

Splenic injury during colonoscopy is rare, but can be fatal (1). A patient with a history of sigmoid colectomy performed for carcinoma of the sigmoid colon four years previously presented for follow-up colonoscopy. It was complicated by a splenic capsular tear resulting in hemoperitoneum; the patient was in shock, and required emergency laparotomy and splenectomy.

During the colonoscopy, the left lateral position was used initially. As difficulty was encountered while negotiating through the splenic flexure, the patient was turned to the supine position, and the procedure was then completed.

Previous abdominal surgery is a reported risk factor for this complication (2). Partial capsular avulsion resulting from looping around the splenic flexure is thought to be the underlying mechanism (3). Positioning of the patient also plays an important role. In the left lateral position (Figure 1), both the splenic flexure and spleen will fall to the left side. Any adhesion band between them will be loose, and the risk of splenic injury is minimized. In the supine position (Figure 2), the spleen will fall towards the back. However, the splenic flexure is tracked towards the opposite direction, either as a result of an attempt to straighten the colonoscopy, with its tip hooked around the splenic flexure, or while the colonoscope is negotiated through the splenic flexure and bringing the splenic flexure with it. Any adhesion band between them will be tightened and splenic capsular tearing can easily occur.

In patients with previous abdominal surgery who are undergoing colonoscopy, the supine position should therefore be

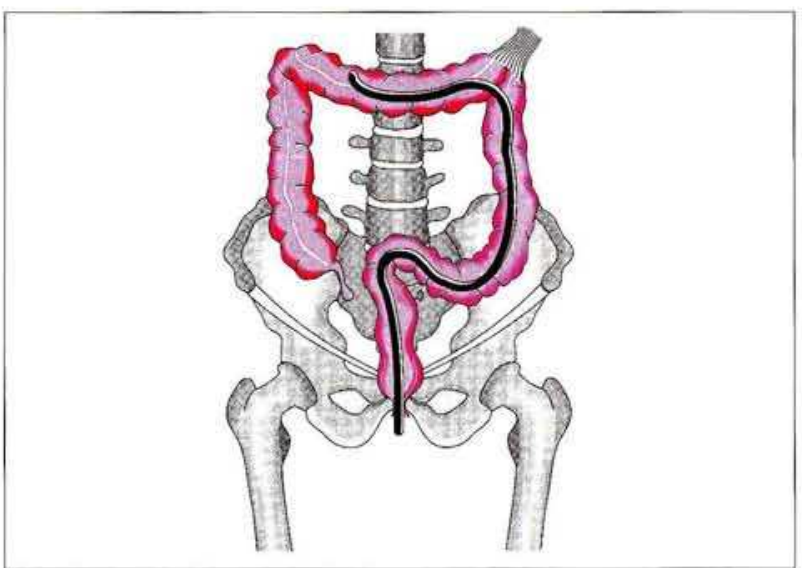

Figure 1: In the left lateral position, both the spleen and the splenic flexure fall to the left side. Any adhesion between them will be lax, and the risk of splenic injury is low

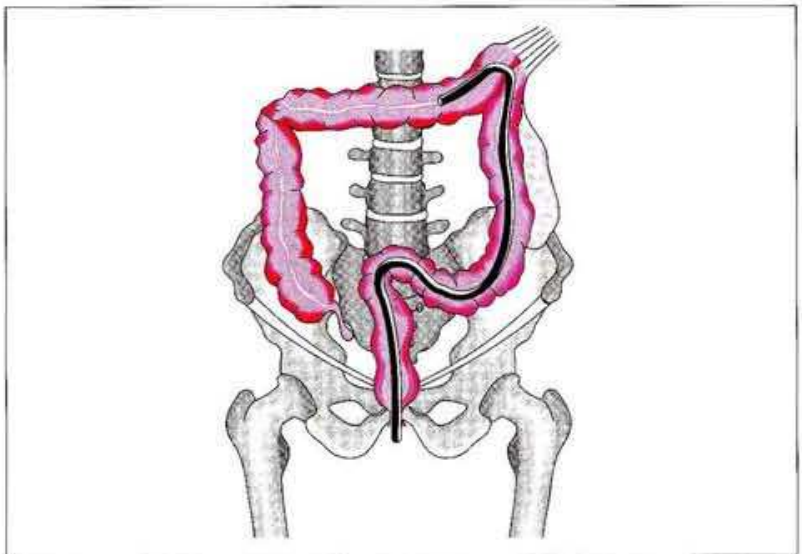

Figure 2: In the supine position, the spleen will fall towards the back, and the splenic flexure is being tracked towards the opposite direction by the colonoscope. Any adhesion between them will be tightened, and this may easily result in splenic injury. 


\section{Unusual Cases and Technical Notes 575}

avoided while negotiating through the splenic flexure, in order to prevent splenic injury.

C. C. W. Tse, K. M. Chung, J. S. T. Hwang Dept. of Surgery, Queen Elizabeth Hospital, Hong Kong
References

1. Espinal EA, Hoak T, Slezak FA. Splenic rupture from colonoscopy. Surg Endosc 1997; 11: 71 -3.

2. Colarian J, Alovski M, Cazzada R. Splenic trauma during colonoscopy. Endoscopy 1991; 23: 48-9.

3. Gores PF, Simso LA. Splenic injury during colonoscopy. Arch Surg 189; 124: 1342 .
Corresponding Author

C. C. W. Tse, M.D.

Block H, 10/F

Dept. of Surgery

Queen Elizabeth Hospital

30 Gascoigne Road

Kowloon

Hong Kong

Fax: + 852-2770-4466 